(2) Open Access Full Text Article

\title{
Exploring the effect of chidamide on blastic plasmacytoid dendritic cell neoplasm: a case report and literature review
}

This article was published in the following Dove Press journal:

Therapeutics and Clinical Risk Management

\author{
Shunan Wang' \\ Wei Guo' \\ Xin Wan' \\ Yongliang Teng ${ }^{2}$ \\ Xiaoge Zhou ${ }^{3}$ \\ Ou Bai' \\ 'Department of Hematology, \\ ${ }^{2}$ Department of Pathology, The \\ First Hospital of Jilin University, \\ Changchun, ${ }^{3}$ Department of Pathology, \\ Beijing Friendship Hospital, Capital \\ Medical University, Beijing, People's \\ Republic of China
}

\begin{abstract}
Blastic plasmacytoid dendritic cell neoplasm (BPDCN) is a rare and aggressive hematopoietic malignancy mainly affecting elderly patients. It is highly responsive to chemotherapy, but the median event-free survival is very short and has a high rate of relapse even after performing allogeneic stem cell transplantation; thus, the discovery of novel agents for the treatment of BPDCN is urgent. Chidamide is a new oral isotype-selective histone deacetylase inhibitor (HDACi). It is proved to exert a well-characterized anticancer property in a wide range of hematological malignancies, especially lymphoma. Here, we report a 41-year-old man who used oral chidamide $30 \mathrm{mg}$ twice per week for maintenance therapy after receiving complete remission. For the first time in this field, we had explored the efficiency of chidamide in the treatment of BPDCN and tried to give more choices to the therapy of this disease.

Keywords: hematopoietic malignancy, skin lesions, maintenance therapy, histone deacetylase inhibitor
\end{abstract}

\section{Introduction}

Blastic plasmacytoid dendritic cell neoplasm (BPDCN) is a rare and aggressive hematopoietic malignancy derived from precursor plasmacytoid dendritic cells. It was classified as a distinct entity among myeloid neoplasms in the 2016 WHO classification. ${ }^{1}$ It mainly affects older individuals with a median age of 60-70 years and has an obvious male predominance. Most patients present with asymptomatic skin lesions as the first symptom and have a high frequency of bone marrow involvement. BPDCN is typically characterized by CD4+ and CD56+ co-expression without common lymphoid or myeloid lineage markers. Accounting for only $0.44 \%$ of all hematological malignancies, the standard and definitive systemic chemotherapy regimen of BPDCN is difficult to establish. ${ }^{2}$ The prognosis of this disease is very poor with a mean survival of 12-14 months. ${ }^{3}$ Thus, it is urgent to illuminate the pathobiology of BPDCN and utilize novel agents in this disease. Chidamide is a new oral isotype-selective histone deacetylase inhibitor (HDACi) that has been proved to be effective in a wide range of hematological malignancies and has been approved in China for the treatment of recurrent or refractory peripheral T-cell lymphoma (PTCL).

In this study, we present the case of a BPDCN patient who underwent central nervous system (CNS) involvement soon after the first complete remission (CR). In the second $\mathrm{CR}$, novel regimen chidamide was chosen to maintain the therapeutic effect. This is the first case of BPDCN treated with chidamide, giving more choice to the clinical application of chidamide. 


\section{Case presentation Diagnosis}

Written informed consent was obtained from the patient's next of kin for publication of this case report and the associated images. A 41-year-old man presenting with an 8-day history of left inguinal lymph node enlargement came to the hospital for evaluation. A physical examination showed palpable left inguinal lymph node measuring 2-4 cm; no skin lesions were identified. The inguinal lymph node biopsy showed effacement of normal nodal architecture by diffuse proliferation of homogeneous midsized lymphoid cells with ovoid or pleomorphic nuclei, small or inconspicuous nucleoli, hypochromic chromatin and red blood cells and small lymphocytes dispersed within them (Figure 1A). Immunohistochemical analysis showed that the neoplastic cells were positive for $\mathrm{CD} 43$, indicating hematopoietic malignancy, and were specifically positive for CD123, CD4 and CD56 (Figure 1B-D) but negative for all other markers examined, including T-cell (CD3, CD2, CD5, CD8), B-cell (CD20, CD79a), myeloid- or monocytic-associated (MPO, CD34) markers. Hence, the pathological diagnosis of BPDCN was made. Ki-67 was positive in $\sim 30 \%-40 \%$ of atypical cells. Bone marrow smear, immunohistochemical analysis of bone marrow biopsy and flow cytometric analysis of bone marrow fluid indicated the involvement of bone marrow. Karyotype analysis revealed chromosomal abnormalities, including 46,XY,t(9;17)(q34;q21) and 46,XY,inv(17) (q21q23). In situ hybridization for the Epstein-Barr virus genome, T-cell gene rearrangement studies and genotyping detection showed no abnormality. With no skin lesions or abnormality on blood cell count, the patient refused immediate therapy.

After 5 months, with progressive diffuse lymphadenopathy, facial skin lesions (Figure 2A), conjunctival congestion and dyspnea on exertion, the patient returned to the hospital for further treatment. Bone marrow smear was performed again, and the specimen demonstrated diffuse infiltration of lymphoblast and prolymphocyte, accounting for 58.5\% compared with $15.5 \%$ last time. Fluorodeoxyglucose positron emission tomography/computed tomography imaging revealed markedly increased fluorodeoxyglucose-avid region in diffuse swollen lymph nodes, enlarged spleen and the whole skeleton. The involvement of diffuse lymph nodes, spleen, skin and bone marrow was confirmed.

This report involves a case of BPDCN with a young patient who presented with specifically facial skin lesions, and the disease was extensively invasive and progressing rapidly.
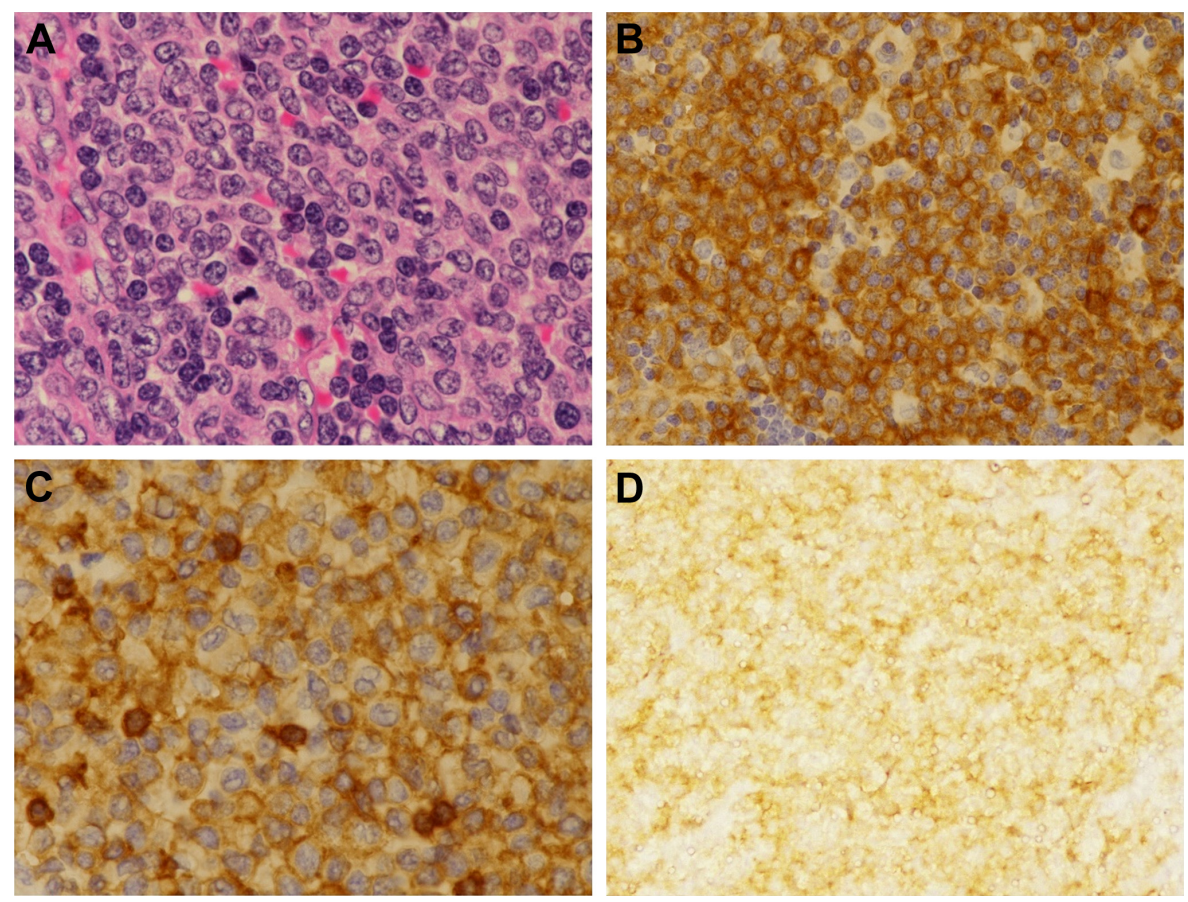

Figure I The results of swollen lymph node biopsy section.

Notes: (A) Diffuse proliferation of homogeneous midsized lymphoid cells with ovoid or pleomorphic nuclei, small or inconspicuous nucleoli and hypochromic chromatin ( $\times 400)$. (B) CDI23 positive for neoplasm cells $(\times 400)$. (C) CD4 positive for neoplasm cells $(\times 400)$. (D) CD56 positive for neoplasm cells $(\times 400)$. 

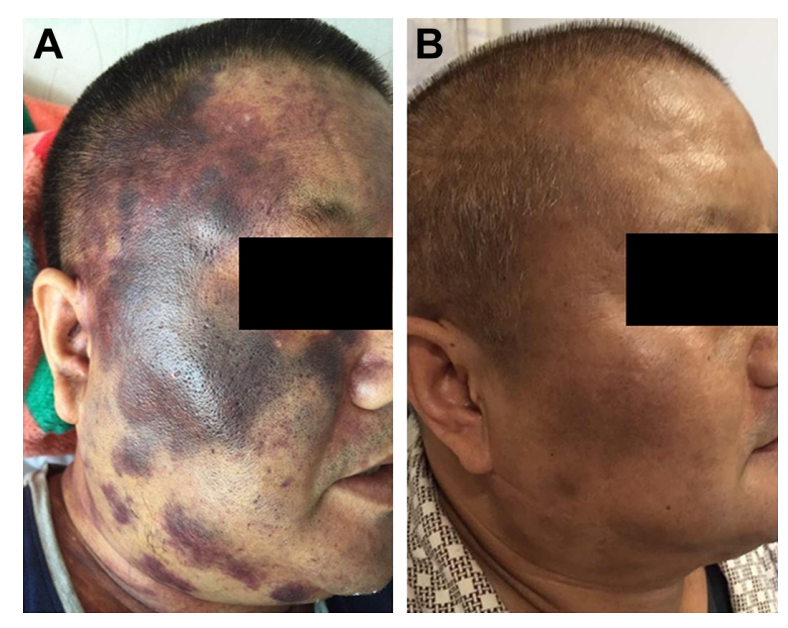

Figure 2 Change in skin lesions.

Notes: (A) Large skin lesions on the face before therapy. (B) Almost disappearance of skin lesions after two cycles of chemotherapy.

\section{Inductive and consolidative therapy}

The process of inductive and consolidative therapy is listed in Table 1. In the first $C R$, there is no lymphadenopathy, no skin lesions (Figure 2B) and no plasmacytoid juvenile cells in the bone marrow or peripheral blood. The patient agreed to receive allogeneic stem cell transplantation (allo-SCT) but no appropriate donor was found. When the patient returned for the third cycle of polychemotherapy, flow cytometry of cerebrospinal fluid suggested aberrant initial cells accounting for $30.72 \%$, indicating the relapse and involvement of the CNS. The second CR was received soon. However, experiencing the high aggressiveness of this disease personally and accounting for the high rate of relapse even after allo-SCT, the patient gave up receiving allo-SCT.
The patient was highly responsive to acute lymphoblastic leukemia (ALL)-like regimens but the involvement of the CNS occurred soon, indicating the importance of CNS prophylaxis. However, regimens used in relapsed/refractory ALL were still effective.

The possible reasons of CNS relapse are as follows: First, CNS involvement occurs in $10 \%$ of all patients with BPDCN at diagnosis and in $30 \%$ of relapsed patients, so the detection of cerebrospinal fluid is crucial to increase the accuracy rate of the diagnosis and the prophylactic intrathecal therapy at the very beginning may help to prevent the CNS relapse. Second, bone marrow involvement was presented in this patient at diagnosis, which indicated that the patient was seriously ill and the disease was highly aggressive with a high rate of relapse. Third, the patient did not receive therapy immediately, and a 5-month delay gave the opportunity for rapid deterioration of the disease.

\section{Maintenance therapy}

Being tired of chemotherapy and wishing for improvement in the quality of life, the patient refused chemotherapy demanding intravenous instillation and chose to prevent disease relapse with oral anticancer medicine, which was well tolerated and effective. After cautious discussion and repeated communication with the patient, a novel regimen involving chidamide was chosen ultimately, and it was taken orally $30 \mathrm{mg}$ twice per week (biw) to prevent the relapse. Blood cell count and biochemical index were tested regularly to monitor the side effects. Chidamide appeared to be well tolerated, and only thrombocytopenia (grade 2) occurred but the platelet levels recovered soon after transitory drug withdrawal.

Table I Inductive and consolidative therapy

\begin{tabular}{|c|c|c|c|c|}
\hline No & Regimen & Dose & Course & Response \\
\hline 1 & VDCP & $\begin{array}{l}\text { CTX I,200 mg/m day I; VDS } 4 \mathrm{mg} \text { days I, } 8, \text { I5, 22; } \\
\text { DNR } 45 \mathrm{mg} / \mathrm{m}^{2} \text { days I-3; PDN } 60 \mathrm{mg} / \mathrm{m}^{2} \text { days I-2I }\end{array}$ & $\mathrm{I}$ & PR \\
\hline 2 & VDCP & $\begin{array}{l}\text { CTX I, } 200 \mathrm{mg} / \mathrm{m}^{2} \text { day I; VDS } 4 \mathrm{mg} \text { days I, 8, I5, 22; } \\
\text { DNR } 45 \mathrm{mg} / \mathrm{m}^{2} \text { days I-3; PDN } 60 \mathrm{mg} / \mathrm{m}^{2} \text { days I-2I }\end{array}$ & I & CNS relapse \\
\hline 3 & $\begin{array}{l}\text { CAM+ intrathecal } \\
\text { injection }\end{array}$ & $\begin{array}{l}\text { CTX } 750 \mathrm{mg} / \mathrm{m}^{2} \text { days I, } 8 ; \text { Ara-C } 100 \mathrm{mg} / \mathrm{m}^{2} \text { days I-3, } \\
\text { 8-10; 6-MP } 60 \mathrm{mg} / \mathrm{m}^{2} \text { days I-7 }\end{array}$ & I & PR \\
\hline 4 & $\begin{array}{l}\text { HD-MTX+ intrathecal } \\
\text { injection }\end{array}$ & MTX $3 \mathrm{~g} / \mathrm{m}^{2}$ day I & I & $C R$ \\
\hline 5 & $\begin{array}{l}\text { Hyper B+ intrathecal } \\
\text { injection }\end{array}$ & $\begin{array}{l}\text { MTX } 1.0 \mathrm{~g} / \mathrm{m}^{2} \text { day } \mathrm{I} ; \text { Ara-C } 2 \mathrm{~g} / \mathrm{m}^{2} \text { days } 2-3 \mathrm{QI} 2 \mathrm{H} \text {; } \\
\text { methylprednisolone } 30 \mathrm{mg} / \mathrm{m}^{2} \text { days I-3 }\end{array}$ & I & $C R$ \\
\hline 6 & $\begin{array}{l}\text { HD-MTX intrathecal } \\
\text { injection }\end{array}$ & MTX $3 \mathrm{~g} / \mathrm{m}^{2}$ day I & I & $\mathrm{CR}$ \\
\hline
\end{tabular}

Abbreviations: 6-MP, 6-mercaptopurine; Ara-C, cytarabine; CAM+, cyclophosphamide, cytarabine, 6-mercaptopurine plus; CNS, central nervous system; CR, complete remission; CTX, cyclophosphamide; DNR, daunorubicin; HD-MTX, high-dose methotrexate; Hyper B+, methotrexate, cytarabine, methylprednisolone plus; MTX, methotrexate; PDN, prednisone; PR, partial remission; QI2H, every 12 hours; VDCP, vindesine, daunorubicin, cyclophosphamide, prednisone; VDS, vindesine. 
Unfortunately, after 2-month therapy of oral chidamide, recurrent skin lesions and bone marrow blasts appeared. The patient relapsed and finally succumbed to the disease 17 months after the initial onset of lymphadenopathy.

\section{Discussion}

BPDCN is extremely rare, and its clinical presentation is often ambiguous and confusing. The atypical immunophenotype also adds difficulty to the precise diagnosis. There is no consensus on the optimal therapeutic strategy for BPDCN. It is highly responsive to chemotherapy used for ALL, acute myeloid leukemia (AML) and non-Hodgkin lymphoma, but the median event-free survival is only 10 months. ${ }^{4}$ Some patients received long-term survival after allo-SCT, especially children, but there were still $30 \%-40 \%$ of them who experienced relapse even after performing allo-SCT. ${ }^{5}$ Hence, the understanding of the pathobiology of BPDCN and the development of novel agents is urgently demanded.

There are very few clinical studies on novel agents about BPDCN. Frankel et $\mathrm{al}^{6}$ reported the first prospective study on the treatment of BPDCN, in which 11 patients were treated with a single course of SL-401 at $12.5 \mu \mathrm{g} / \mathrm{kg}$ intravenously over 15 minutes daily for up to five doses. Seven of nine evaluable (78\%) BPDCN patients had major responses, including five complete responses and two partial responses after a single course of SL-401. The median duration of response (DOR) was 5 months (range, 1-20+ months). Montero et $\mathrm{al}^{7}$ reported the use of venetoclax, a kind of BCL-2 inhibitor, in two patients with relapsed/refractory BPDCN. Both of them received a daily dose escalation of venetoclax of $50 \mathrm{mg}, 100 \mathrm{mg}$ and $200 \mathrm{mg}$ to a final dose of $400 \mathrm{mg}$ daily, and the significant decrease in tumor burden was observed at the fourth weeks. Gemcitabine, a well-known nucleoside analog, was found to be efficient in an 80-yearold patient with $\mathrm{BPDCN}{ }^{8}$ Ulrickson et $\mathrm{al}^{9}$ reported three patients with relapsed BPDCN who received the gemcitabine/ docetaxel regimen (gemcitabine $800 \mathrm{mg} / \mathrm{m}^{2}$ days 1 and 8 and docetaxel $75 \mathrm{mg} / \mathrm{m}^{2}$ day 8 every 3 weeks); the median overall survival (OS) was 13.3 months (range 8-17 months) with one patient remaining alive and in remission 15 months after treatment. Bétrian et $\mathrm{al}^{10}$ reported five patients treated with bendamustine hydrochloride, a well-tolerated bifunctional drug acting as an alkylating and antimetabolite agent. Among the four patients evaluable for response, two failed therapy, one died from tumor lysis syndrome after rapid blast clearance from blood and one reached and maintained CR for 7 months, suggesting that bendamustine should be further evaluated in BPDCN.
Chidamide, the first listed benzamide class of HDACi in the world, is currently undergoing global clinical trials for solid tumor treatments. ${ }^{11}$ Numerous studies reveal that chidamide exerts a well-characterized anticancer property in a wide range of hematological malignancies, for example, lymphoma, myeloma, AML and ALL. ${ }^{12-15}$ Chidamide and therapeutic chemotherapy have a cooperative effect of inducing apoptosis by DNA damage accumulation and repair defects in AML stem and progenitor cells. ${ }^{12}$ The mechanism is similar to the synergistic effect of chidamide and low-dose decitabine in ALL. ${ }^{13}$ At the same time, BPDCN has already been classified under myeloid neoplasms, and chemotherapy regimens for both ALL and AML are efficient in BPDCN. In addition, accounting for the demand of the patient to be treated with oral, well-tolerated, effective anticancer medicine, chidamide was chosen for maintenance therapy. Since chidamide at $30 \mathrm{mg}$ biw has been proved to be perfectly effective and well tolerated in recurrent or refractory PTCL, it was given at $30 \mathrm{mg}$ biw, and the actual side effects were really controllable.

However, the patient soon relapsed and the effect of maintenance therapy was not ideal. Chidamide has been proved to be efficient in acute leukemia when combined with other regimens; hence, chidamide in combination with chemotherapy may be a better therapeutic choice. The patient passed away 17 months after the diagnosis, which was much longer than median OS reported by previous studies about BPDCN and any of the novel agents reported before.

\section{Conclusion}

Here, we report the first case of BPDCN receiving chidamide as maintenance therapy and review clinical studies on the use of novel agents in BPDCN, suggesting that novel agents (SL-401, venetoclax, gemcitabine, docetaxel) can control symptoms and improve DOR and OS. Apart from imaging examination, cerebrospinal fluid should be investigated at diagnosis to identify whether the CNS involvement exists or not and prophylactic intrathecal therapy should be given at the very beginning to prevent the CNS involvement. Chidamide monotherapy in BPDCN demands further investigation, and patients may benefit more when chidamide is used in combination with other regimens. Combination of novel agents with chemotherapy may be the focus of future research on BPDCN.

\section{Acknowledgment}

This study was supported by the Science and Technology Agency of Jilin province, the study on the diversity and 
specificity of lymphoma-associated genes and the individualized treatment of lymphoma (Bethune subject; No 20160101010JC).

\section{Disclosure}

The authors report no conflicts of interest in this work.

\section{References}

1. Julia F, Dalle S, Duru G, et al. Blastic plasmacytoid dendritic cell neoplasms: clinic immunohistochemical correlations in a series of 91 patients. Am J Surg Pathol. 2014;38(5):673-680.

2. Bueno C, Almeida J, Lucio P, et al. Incidence and characteristics of CD4(+)/HLA DRhi dendritic cell malignancies. Haematologica. 2004; 89(1):58-69.

3. Arber DA, Orazi A, Hasserjian R, et al. The 2016 revision to the World Health Organization classification of myeloid neoplasms and acute leukemia. Blood. 2016;127(20):2391-2405.

4. Kim MJ, Nasr A, Kabir B, et al. Pediatric blastic plasmacytoid dendritic cell neoplasm: a systematic literature review. J Pediatr Hematol Oncol. 2017;39(7):528-537.

5. Roos-Weil D, Dietrich S, Boumendil A, et al. Stem cell transplantation can provide durable disease control in blastic plasmacytoid dendritic cell neoplasm: a retrospective study from the European Group for Blood and Marrow Transplantation. Blood. 2013;121(3):440-446.

6. Frankel AE, Woo JH, Ahn C, et al. Activity of SL-401, a targeted therapy directed to interleukin-3 receptor, in blastic plasmacytoid dendritic cell neoplasm patients. Blood. 2014;124(3):385-392.
7. Montero J, Stephansky J, Cai T, et al. Blastic plasmacytoid dendritic cell neoplasm is dependent on BCL2 and sensitive to venetoclax. Cancer Discov. 2017;7(2):156-164.

8. Shepro D, Miller M. Gemcitabine in blastic plasmacytoid dendritic cell neoplasm (CD41CD561 hematodermic neoplasm). Blood. 2014; 124(21):Abstract5457.

9. Ulrickson ML, Puri A, Lindstrom S, et al. Gemcitabine and docetaxel as a novel treatment regimen for blastic plasmacytoid dendritic cell neoplasm. Am J Hematol. 2017;92(5):E75-E77.

10. Bétrian S, Guenounou S, Luquet I, et al. Bendamustine for relapsed blastic plasmacytoid dendritic cell leukaemia. Hematol Oncol. 2017; 35(2):252-255.

11. Gao S, Li X, Zang J, Xu W, Zhang Y. Preclinical and clinical studies of chidamide (CS055/HBI-8000), an orally available subtype-selective HDAC inhibitor for cancer therapy. Anticancer Agents Med Chem. 2017; 17(6):802-812.

12. Li Y, Wang Y, Zhou Y, et al. Cooperative effect of chidamide and chemotherapeutic drugs induce apoptosis by DNA damage accumulation and repair defects in acute myeloid leukemia stem and progenitor cells. Clin Epigenetics. 2017;9(1):83.

13. Shi $\mathrm{P}$, Zhang L, Chen K, et al. Low-dose decitabine enhances chidamideinduced apoptosis in adult acute lymphoblast leukemia, especially for p16-deleted patients through DNA damage. Pharmacogenomics. 2017;18(13):1259-1270.

14. Shi Y, Dong M, Hong X, et al. Results from a multicenter, open-label, pivotal phase II study of chidamide in relapsed or refractory peripheral T-cell lymphoma. Ann Oncol. 2015;26(8):1766-1771.

15. Xu L, Tang HL, Gong X, et al. Inducing effect of chidamide on apoptosis of multiple myeloma cells and its relerance to DNA damage response. Zhongguo Shi Yan Xue Ye Xue Za Zhi. 2015;23(2):450-454.
Therapeutics and Clinical Risk Management

\section{Publish your work in this journal}

Therapeutics and Clinical Risk Management is an international, peerreviewed journal of clinical therapeutics and risk management, focusing on concise rapid reporting of clinical studies in all therapeutic areas, outcomes, safety, and programs for the effective, safe, and sustained use of medicines. This journal is indexed on PubMed Central, CAS,

\section{Dovepress}

EMBase, Scopus and the Elsevier Bibliographic databases. The manuscript management system is completely online and includes a very quick and fair peer-review system, which is all easy to use. Visit http://www.dovepress.com/testimonials.php to read real quotes from published authors. 